Article

\title{
Palladium(II)/Cationic 2,2'-Bipyridyl System as a Highly Efficient and Reusable Catalyst for the Mizoroki-Heck Reaction in Water
}

\author{
Shao-Hsien Huang, Jun-Rong Chen and Fu-Yu Tsai * \\ Institute of Organic and Polymeric Materials, National Taipei University of Technology, 1, Section 3, \\ Chung-Hsiao E. Rd., Taipei 106, Taiwan
}

* Author to whom correspondence should be addressed; E-Mail: fuyutsai@ntut.edu.tw.

Received: 18 December 2009; in revised form: 8 January 2009 / Accepted: 12 January 2010 /

Published: 12 January 2010

\begin{abstract}
A water-soluble and air-stable $\mathrm{Pd}\left(\mathrm{NH}_{3}\right)_{2} \mathrm{Cl}_{2}$ /cationic 2,2'-bipyridyl system was found to be a highly-efficient and reusable catalyst for the coupling of aryl iodides and alkenes in neat water using $\mathrm{Bu}_{3} \mathrm{~N}$ as a base. The reaction was conducted at $140{ }^{\circ} \mathrm{C}$ in a sealed tube in air with a catalyst loading as low as $0.0001 \mathrm{~mol} \%$ for the coupling of activated aryl iodides with butyl and ethyl acrylates, providing the corresponding products in good to excellent yields with very high turnover numbers. In the case of styrene, Mizoroki-Heck coupling products were obtained in good to high yields by using a greater catalyst loading ( $1 \mathrm{~mol} \%)$ and $\mathrm{TBAB}$ as a phase-transfer agent. After extraction, the residual aqueous solution could be reused several times with only a slight decrease in its activity, making the Mizoroki-Heck reaction "greener".
\end{abstract}

Keywords: Mizoroki-Heck reaction; water-soluble catalyst; reusable; cross-coupling

\section{Introduction}

Palladium-catalyzed coupling of aryl halides and alkenes, known as the Mizoroki-Heck reaction, is one of the most powerful processes available in organic synthesis for carbon-carbon bond formation $[1,2]$. This reaction is greatly facilitated in polar aprotic solvents such as DMA, DMF, DMSO, MeCN, and NMP under homogeneous catalysis (for recent reviews, see refs. [3-6]). However, the catalyst and organic products are often of similar solubility in organic solvents, and hence it is difficult to separate 
the catalyst from the reaction mixture and recycle it at the end of the reaction, leading to wastage of precious metals. Because water is an organic immiscible solvent, the introduction of a water-soluble ligand to combine with transition-metal catalysts and use of water as the reaction medium is one of the best methods of overcoming this problem (for recent reviews, see refs. [7-14]), although the reaction rates of organic reactions in water may be slower than in organic solvents due to the low effective concentration of the organic substrate in the aqueous phase. The water-soluble catalytic system may be easily separated from the water-insoluble organic products by simple filtration or extraction, leading to the possibility of reuse of the catalyst. Therefore, the development of a water-compatible and reusable catalytic system is highly attractive and valuable from the green chemistry and economics viewpoints.

There are some reports of the Mizoroki-Heck reaction being performed in neat water, which include reactions in the presence of phase-transfer agents [15-19] and the use of hydrophilic ligands such as phosphines [20,21], oxime derivatives [22], oligoether-substituted benzimidazolim salts [23] and anionic $\mathrm{N}$-donor ligands [24]. Other methods involving the use of supported materials [25-31] and Pdnanoparticles [32-34] as heterogeneous catalysts also allow the reaction to be conducted in the aqueous phase. We have recently prepared water-soluble cationic 2,2'-bipyridyl ligand 1 and utilized it to bring a palladium complex into the aqueous phase for carbon-carbon bond-forming reactions [35-37], a rhodium complex for phenylacetylene polymerization [38], and an iron salt for $S$-arylation [39] under aerobic conditions. As part of our continuing efforts in the development of green and reusable catalytic systems for carbon-carbon bond-forming reactions, we report herein the combination of $\mathrm{Pd}\left(\mathrm{NH}_{3}\right)_{2} \mathrm{Cl}_{2}$ and $\mathbf{1}$ to create a highly-efficient and reusable catalyst for the coupling of aryl iodides and alkenes in water under air, making the Mizoroki-Heck reaction green and economically viable (Scheme 1).

Scheme 1. The Mizoroki-Heck reaction in water.

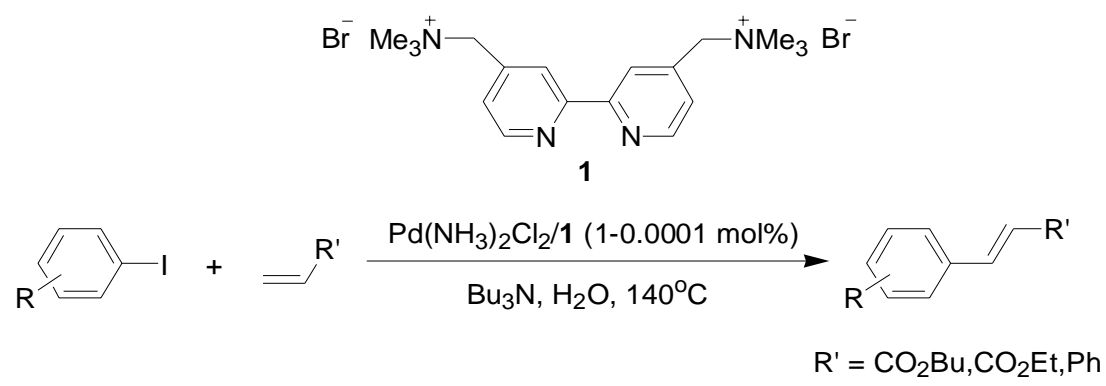

\section{Results and Discussion}

The catalytic system was prepared by mixing equimolar amounts of $\mathrm{Pd}\left(\mathrm{NH}_{3}\right)_{2} \mathrm{Cl}_{2}$ and $\mathbf{1}$ in water. The catalyst is stable in water and can be stored under air; hence, stock solutions of this catalytic system could be prepared at different concentrations. In preliminary studies, our goal was to find the best base for use in the Mizoroki-Heck reaction in water (Table 1). We found that inorganic bases were ineffective for the coupling of iodobenzene 2a and butyl acrylate 3a, even at a reaction temperature of $140{ }^{\circ} \mathrm{C}$ (Entries 1-6). Replacing inorganic bases by organic amines such as $\mathrm{Et}_{3} \mathrm{~N}$ led to the formation of trans-cinnamic acid $n$-butyl ester 4 a in a $32 \%$ yield (Entry 7 ). Among the amines used, the use of 2 equiv. of $\mathrm{Bu}_{3} \mathrm{~N}$ provided the best results, and a near quantitative yield was obtained (Entry 10). The blank reaction was also examined under identical conditions, and no desired product 
was found in the absence of the catalyst, which indicates that the reaction is indeed catalyzed by the Pd system at a very low catalytic concentration. In the absence of $\mathbf{1}$, a yield of only $34 \%$ was obtained, suggesting the importance of the use of a water-soluble ligand for reaction in water. Thus, the optimal reaction conditions were obtained and are shown in entry 10 of Table 1.

Table 1. Base screening for the Mizoroki-Heck reaction of iodobenzene 2a and butyl acrylate 3a in water. ${ }^{a}$

\begin{tabular}{|c|c|c|}
\hline Entry & Base (equiv.) & Yield (\%) \\
\hline 1 & $\mathrm{NaOH}(2)$ & NR \\
\hline 2 & $\mathrm{NaHCO}_{3}(2)$ & 2 \\
\hline 3 & $\mathrm{KOH}(2)$ & NR \\
\hline 4 & KOAc (2) & NR \\
\hline 5 & $\mathrm{~K}_{2} \mathrm{CO}_{3}(2)$ & NR \\
\hline 6 & $\mathrm{KF}(2)$ & 4 \\
\hline 7 & $\mathrm{Et}_{3} \mathrm{~N}(2)$ & 32 \\
\hline 8 & Diisopropylamine (2) & 17 \\
\hline 9 & $N, N$-Diisopropylethylamine (2) & 6 \\
\hline 10 & $\mathrm{Bu}_{3} \mathrm{~N}(2)$ & 99 \\
\hline 11 & $\mathrm{Bu}_{3} \mathrm{~N}(1)$ & 49 \\
\hline $12^{\mathrm{c}}$ & $\mathrm{Bu}_{3} \mathrm{~N}(2)$ & NR \\
\hline $13^{\mathrm{d}}$ & $\mathrm{Bu}_{3} \mathrm{~N}(2)$ & 34 \\
\hline
\end{tabular}

Using the optimal conditions, we next investigated the scope of the reaction of aryl iodides with butyl acrylate (Table 2). Iodobenzene and its derivatives with electron-withdrawing groups at the para-position afforded very high activities, offering the corresponding products, $\mathbf{4 b}-\mathbf{4 f}$, in excellent yields (Entries 2, 4, 6, 8, and 10, respectively). Further reduction of the catalyst loading to $0.0001 \mathrm{~mol} \%$ gave the products at yields between $48 \%$ and $92 \%$ (Entry 1, 3, 5, 7, 9, and 11), and the turnover number (TON) was up to 920,000 (Entry 3). A longer reaction time was required for aryl iodides bearing an electron-donating group at the para-position. Hence, good to high yields could be isolated in $48 \mathrm{~h}$ with a catalyst loading of $0.01 \mathrm{~mol} \%$ (Entries 12-15). Similarly, in the cases of entries $12-15$, both meta- and ortho-substituted aryl iodides gave excellent isolated yields under identical conditions (Entries 16-18).

Although the boiling point of ethyl acrylate $\mathbf{3 b}$ is lower than the reaction temperature, the MizorokiHeck coupling of aryl iodides with $\mathbf{3 b}$ still proceeded smoothly in a sealed tube. As shown in Table 3, the corresponding products were isolated in yields slightly lower than that of the coupling with butyl acrylate under similar conditions (Entries 1-17). The catalyst loading for the reaction of activated aryl iodides with $\mathbf{3 b}$ could also be further reduced to $0.0001 \mathrm{~mol} \%$, which led to the formation of the desired products in good to high yields with a very high TON (Entries 2, 4, 6, 8, 10, and 12). 
Table 2. Mizoroki-Heck reaction of aryl iodides 2a-1 and butyl acrylate 3a in water. ${ }^{a}$

\begin{tabular}{|c|c|c|c|c|c|c|c|}
\hline Entry & Aryl iodide & & $\begin{array}{ll}\text { Pd/1 } & \text { (mol } \\
\%) & \\
\end{array}$ & Time (h) & Product $(\%)^{\mathbf{b}}$ & & TON \\
\hline $1^{c}$ & & $2 a$ & 0.0001 & 48 & $\mathrm{CO}_{2} \mathrm{Bu}$ & 4a (48) & 480,000 \\
\hline 2 & & $2 \mathbf{b}$ & 0.01 & 12 & $\mathrm{CO}_{2} \mathrm{Bu}$ & 4b (99) & 9,900 \\
\hline $3^{c}$ & & $2 \mathbf{b}$ & 0.0001 & 48 & & $\mathbf{4 b}(92)$ & 920,000 \\
\hline 4 & & 2c & 0.01 & 12 & $-\mathrm{CO}_{2} \mathrm{Bu}$ & 4c (99) & 9,900 \\
\hline $5^{c}$ & & 2c & 0.0001 & 48 & & 4c $(80)$ & 800,000 \\
\hline 6 & & 2d & 0.01 & 12 & $-\mathrm{CO}_{2} \mathrm{Bu}$ & 4d (99) & 9,900 \\
\hline $7^{c}$ & & 2d & 0.0001 & 48 & & 4d (86) & 860,000 \\
\hline 8 & & $2 e$ & 0.01 & 12 & $-\mathrm{CO}_{2} \mathrm{Bu}$ & $\mathbf{4 e}(91)$ & 9,100 \\
\hline $9^{c}$ & & $2 e$ & 0.0001 & 48 & Eto & 4e (68) & 680,000 \\
\hline 10 & & $2 f$ & 0.01 & 12 & $-\mathrm{CO}_{2} \mathrm{Bu}$ & 4f $(92)$ & 9,200 \\
\hline $11^{\mathrm{c}}$ & & $2 f$ & 0.0001 & 48 & & 4f $(91)$ & 910,000 \\
\hline 12 & & $2 g$ & 0.01 & 12 & $-\mathrm{CO}_{2} \mathrm{Bu}$ & $4 g(67)$ & 6,700 \\
\hline 13 & & $2 g$ & 0.01 & 48 & & $4 \mathbf{g}(94)$ & 9,400 \\
\hline 14 & & $2 h$ & 0.01 & 48 & & 4h (92) & 9,200 \\
\hline 15 & $-\mathrm{OH}$ & $2 \mathbf{i}$ & 0.01 & 48 & & $4 \mathbf{i}(56)$ & 5,600 \\
\hline 16 & & $2 \mathbf{j}$ & 0.01 & 48 & & $\mathbf{4 j}(96)$ & 9,600 \\
\hline 17 & & $2 \mathbf{k}$ & 0.01 & 48 & & $4 \mathbf{k}(95)$ & 9,500 \\
\hline 18 & & 21 & 0.01 & 48 & & $4 \mathbf{l}(96)$ & 9,600 \\
\hline
\end{tabular}

${ }^{\mathrm{a}}$ Reaction conditions: aryl iodide $(1 \mathrm{mmol})$, butyl acrylate $(1.5 \mathrm{mmol}), \mathrm{Bu}_{3} \mathrm{~N}(2 \mathrm{mmol})$, $\mathrm{Pd}\left(\mathrm{NH}_{3}\right)_{2} \mathrm{Cl} / \mathbf{1}(0.01$ or $0.0001 \mathrm{~mol} \%)$, and $\mathrm{H}_{2} \mathrm{O}(3 \mathrm{~mL})$ at $140{ }^{\circ} \mathrm{C}$; ${ }^{\mathrm{b}}$ Isolated yields. ${ }^{\mathrm{c}}$ Ten mmol of aryl iodide was used.

Table 3. Mizoroki-Heck reaction of aryl iodides $\mathbf{2 a - k}$ and ethyl acrylate $3 \mathbf{b}$ in water. ${ }^{\text {a }}$

\begin{tabular}{|c|c|c|c|c|c|c|c|}
\hline Entry & Aryl iodide & & Pd/1 (mol \%) & Time (h) & Product $(\%)^{b}$ & & TON \\
\hline 1 & & $2 a$ & 0.01 & 12 & $-\mathrm{CO}_{2} \mathrm{Et}$ & $5 a(63)$ & 6,300 \\
\hline $2^{c}$ & & $2 a$ & 0.0001 & 48 & & $5 a(58)$ & 580,000 \\
\hline 3 & & $2 \mathbf{b}$ & 0.01 & 12 & $\mathrm{CO}_{2} \mathrm{Et}$ & $5 \mathbf{b}(82)$ & 8,200 \\
\hline $4^{\mathrm{c}}$ & & $2 \mathbf{b}$ & 0.0001 & 48 & & $5 \mathbf{b}(81)$ & 810,000 \\
\hline 5 & & 2c & 0.01 & 12 & & $5 c(74)$ & 7,400 \\
\hline $6^{\mathrm{c}}$ & & 2c & 0.0001 & 48 & & $5 c(51)$ & 510,000 \\
\hline 7 & & 2d & 0.01 & 12 & $-\mathrm{CO}_{2} \mathrm{Et}$ & $5 \mathbf{d}(80)$ & 8,000 \\
\hline $8^{c}$ & & 2d & 0.0001 & 48 & & $5 d(76)$ & 760,000 \\
\hline 9 & & $2 e$ & 0.01 & 12 & $-\mathrm{CO}_{2} \mathrm{Et}$ & $5 \mathbf{e}(81)$ & 8,100 \\
\hline $10^{\mathrm{c}}$ & & $2 e$ & 0.0001 & 48 & Eto & $5 e(45)$ & 450,000 \\
\hline
\end{tabular}


Table 3. Cont.

\begin{tabular}{|c|c|c|c|c|c|c|c|}
\hline Entry & Aryl iodide & & $\begin{array}{ll}\mathrm{Pd} / \mathbf{1} & \text { (mol } \\
\% & \\
\end{array}$ & Time (h) & Product $(\%)^{b}$ & & TON \\
\hline 11 & & $2 f$ & 0.01 & 12 & $-\mathrm{CO}_{2} \mathrm{Et}$ & $5 \mathbf{f}(82)$ & 8,200 \\
\hline $12^{\mathrm{c}}$ & & $2 f$ & 0.0001 & 48 & & $5 f(81)$ & 810,000 \\
\hline 13 & -OMe & $2 \mathrm{~g}$ & 0.01 & 48 & & $5 g(82)$ & 8,200 \\
\hline 14 & & $2 h$ & 0.01 & 48 & & $5 h(74)$ & 7,400 \\
\hline 15 & ) $\mathrm{OH}$ & $2 \mathbf{i}$ & 0.01 & 48 & & $5 \mathbf{i}(44)$ & 4,400 \\
\hline 16 & & $2 \mathbf{j}$ & 0.01 & 48 & & $5 \mathbf{j}(59)$ & 5,900 \\
\hline 17 & $M$ & $2 k$ & 0.01 & 48 & & $5 \mathbf{k}(65)$ & 6,500 \\
\hline
\end{tabular}

${ }^{\mathrm{a}}$ Reaction conditions: aryl iodide $(1 \mathrm{mmol})$, ethyl acrylate $(1.5 \mathrm{mmol}), \mathrm{Bu}_{3} \mathrm{~N}(2 \mathrm{mmol})$, $\mathrm{Pd}\left(\mathrm{NH}_{3}\right)_{2} \mathrm{Cl} / \mathbf{1}(0.01$ or $0.0001 \mathrm{~mol} \%)$, and $\mathrm{H}_{2} \mathrm{O}(3 \mathrm{~mL})$ at $140{ }^{\circ} \mathrm{C} ;{ }^{b}$ Isolated yields; ${ }^{\mathrm{c}}$ Ten mmol of aryl iodide was used.

The reaction rate of iodobenzene with styrene under this catalytic system was much slower than that of butyl and ethyl acrylate, providing only $36 \%$ of $\mathbf{6 a}$ when 1 mol \% of catalyst was employed (Table 4, Entry 1). The addition of a phase-transfer agent, TBAB, to the mixture improved the reaction efficiently to give $\mathbf{6 a}$ in an $89 \%$ yield (Entry 2 ). Thus, TBAB was added when styrene was used as the reactant. Under such conditions, aryl iodides with not only electron-withdrawing but also electrondonating groups at the para-position could couple with styrene efficiently to afford the corresponding trans-stilbene derivatives, 6b-6i, in high yields (Entries 3-9). In addition, 3-iodotoluene could also react with styrene to furnish $\mathbf{6 j}$ in a $68 \%$ yield (Entry 10), and even sterically-hindered aryl iodides, 2k-2m, were able to be coupled efficiently under this system, the corresponding products being obtained at yields between $73 \%$ and $93 \%$ at $140{ }^{\circ} \mathrm{C}$ within $24 \mathrm{~h}$ (Entries $11-13$ ).

Table 4. Mizoroki-Heck reaction of aryl iodides 2a-m and styrene 3c in water. ${ }^{a}$

\begin{tabular}{|c|c|c|c|c|c|c|}
\hline Entry & Aryl iodide & & Pd/1 (mol \%) & TBAB (equiv) & Product $(\%)^{b}$ & \\
\hline 1 & & $2 a$ & 1 & 0 & & 6a (36) \\
\hline 2 & & $2 a$ & 1 & 1 & & 6a (89) \\
\hline 3 & 1) $-\mathrm{come}$ & $2 b$ & 1 & 1 & & $\mathbf{6 b}(93)$ \\
\hline 4 & $-\mathrm{NO}_{2}$ & 2c & 1 & 1 & & 6c $(82)$ \\
\hline 5 & & 2d & 1 & 1 & & $\mathbf{6 d}(83)$ \\
\hline 6 & $-\mathrm{CO}_{2} \mathrm{Et}$ & $2 e$ & 1 & 1 & & $\mathbf{6 e}(76)$ \\
\hline 7 & $\longrightarrow-\mathrm{Cl}$ & $2 f$ & 1 & 1 & & $\mathbf{6 f}(87)$ \\
\hline
\end{tabular}


Table 4. Cont.

\begin{tabular}{|c|c|c|c|c|c|c|}
\hline Entry & Aryl iodide & & $\begin{array}{c}\mathrm{Pd} / \mathbf{1}(\mathrm{mol} \\
\%) \\
\end{array}$ & TBAB (equiv) & Product (\%)b & \\
\hline 8 & -OMe & $2 g$ & 1 & 1 & & $6 \mathbf{g}(82)$ \\
\hline 9 & & $2 h$ & 1 & 1 & & 6h $(71)$ \\
\hline 10 & $-\mathrm{OH}$ & $2 \mathbf{i}$ & 1 & 1 & & $6 \mathbf{i}(69)$ \\
\hline 11 & & $2 \mathbf{j}$ & 1 & 1 & & $\mathbf{6 j}(68)$ \\
\hline 12 & & $2 \mathbf{k}$ & 1 & 1 & & $\mathbf{6 k}(73)$ \\
\hline 13 & & 21 & 1 & 1 & & $61(93)$ \\
\hline 14 & & $2 m$ & 1 & 1 & & 6m (84) \\
\hline
\end{tabular}

${ }^{\mathrm{a}}$ Reaction conditions: aryl iodide $(1 \mathrm{mmol})$, styrene $(1.5 \mathrm{mmol}), \mathrm{Bu}_{3} \mathrm{~N}(2 \mathrm{mmol}), \mathrm{Pd}\left(\mathrm{NH}_{3}\right)_{2} \mathrm{Cl} / \mathbf{1}$ (1 mol \%), TBAB (1 mmol), and $\mathrm{H}_{2} \mathrm{O}(3 \mathrm{~mL})$ at $140{ }^{\circ} \mathrm{C}$ for $24 \mathrm{~h} ;{ }^{\mathrm{b}}$ Isolated yields.

As for the coupling of cheaper aryl bromides, we found that only activated aryl bromides could couple with butyl acrylate to afford the corresponding Mizoroki-Heck coupling products in good yields with $1 \mathrm{~mol} \%$ catalyst in the presence of 0.5 equiv TBAB. The use of bromobenzene and a deactivated aryl bromide such as 4-bromoanisole did not furnish any desired product (Scheme 2).

Scheme 2. The Mizoroki-Heck reaction of aryl bromides with butyl acrylate in water.

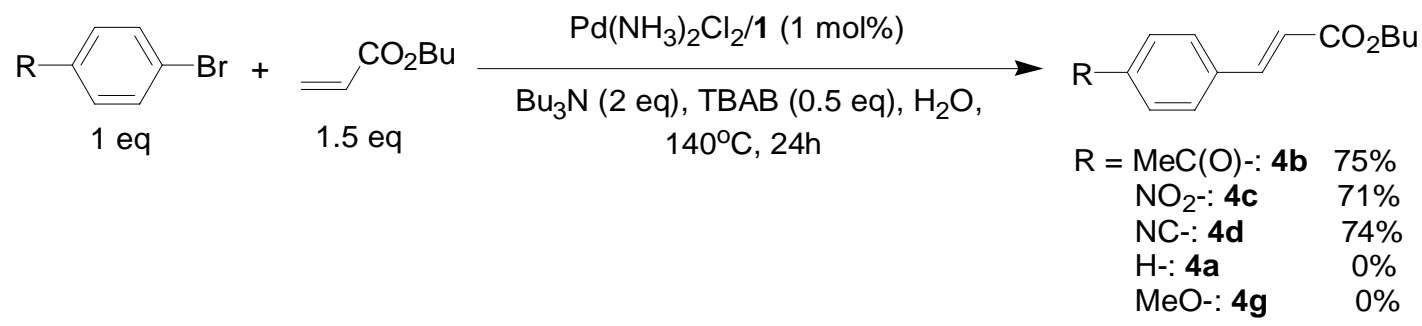

We then examined the reusability of the residual aqueous solution, which is important from the viewpoints of practical utilization and economics. We employed butyl and ethyl acrylates and styrene individually to react with 4-iodoacetophone, $\mathbf{2 b}$, to test the reusability of this catalytic system (Table 5). The Mizoroki-Heck coupling of butyl acrylate and $\mathbf{2 b}$ with a $0.01 \mathrm{~mol} \%$ catalyst loading led to the formation of $\mathbf{4 b}$ in a $99 \%$ yield in $12 \mathrm{~h}$. After completion of the first cycle, hexane was used to extract the organic product and the residual aqueous solution was recharged with $\mathrm{Bu}_{3} \mathrm{~N}$, butyl acrylate, and 4-iodoacetophone for the second cycle. It was found that the residual aqueous solution could be reused at least four times with only a slight decrease in activity (Entry 1). The employment of ethyl acrylate led to a similar reusability with slightly lower yields as compared with butyl acrylate (Entry 2). In the case of styrene, a higher catalyst loading (1 mol \%) and TBAB were employed in the 
initial cycle in order to lead to a higher product yield. Addition of the phase-transfer agent in subsequent runs was not required (Entry 3).

Table 5. Reuse studies of the Mizoroki-Heck reaction of 4-iodoacetophone $\mathbf{2 b}$ and alkenes $\mathbf{3}$. ${ }^{\mathrm{a}}$

\begin{tabular}{|c|c|c|c|c|c|c|c|c|c|}
\hline \multirow{2}{*}{ Entry } & \multirow{2}{*}{ Alkene } & & \multirow{2}{*}{ Time (h) } & \multirow{2}{*}{$\begin{array}{l}\mathrm{Pd} / \mathbf{1} \\
\%)\end{array}$} & \multicolumn{5}{|c|}{ Cycle (\%) } \\
\hline & & & & & 1st & 2nd & 3rd & 4th & 5th \\
\hline 1 & $\mathrm{CO}_{2} \mathrm{Bu}$ & 3a & 12 & 0.01 & 99 & 98 & 95 & 93 & 90 \\
\hline 2 & $-\mathrm{CO}_{2} \mathrm{Et}$ & $3 b$ & 12 & 0.01 & 90 & 90 & 86 & 83 & 81 \\
\hline $3^{c}$ & $\mathrm{Ph}$ & $3 c$ & 24 & 1 & 93 & 94 & 93 & 92 & 90 \\
\hline
\end{tabular}

${ }^{\mathrm{a}}$ Reaction conditions: 4-iodoacetophone (1 mmol), alkene (1.5 mmol), $\mathrm{Bu}_{3} \mathrm{~N}$ (2 mmol), $\mathrm{Pd}\left(\mathrm{NH}_{3}\right)_{2} \mathrm{Cl} / \mathbf{1}$ (1 or $0.01 \mathrm{~mol} \%$ ), and $\mathrm{H}_{2} \mathrm{O}(3 \mathrm{~mL})$ at $140{ }^{\circ} \mathrm{C}$; ${ }^{\mathrm{b}}$ Isolated yields; ${ }^{\mathrm{c}}$ One mmol of TBAB was added.

\section{Experimental}

\subsection{General}

Chemicals were purchased from commercial suppliers and were used without further purification. The cationic 2,2'-bipyridyl ligand was prepared according to the published procedures [24,25]. GC analysis was performed on a Shimadzu GC-14B equipped with a fused silica capillary column, and all ${ }^{1} \mathrm{H}$ - and ${ }^{13} \mathrm{C}$-NMR spectra were recorded in $\mathrm{CDCl}_{3}$ at $25{ }^{\circ} \mathrm{C}$ on a Varian $200 \mathrm{NMR}$ spectrometer. Elemental analyses were performed and high resolution mass spectra recorded at the Instrument Center Service, National Science Council of Taiwan.

\subsection{Typical procedure for the Mizoroki-Heck reaction}

A sealable tube equipped with a magnetic stirrer bar was charged with aryl iodide (1 mmol), alkene (1.5 mmol), $\mathrm{Bu}_{3} \mathrm{~N}(2 \mathrm{mmol})$, and $\mathrm{H}_{2} \mathrm{O}(2 \mathrm{~mL})$. In the case of styrene, the addition of TBAB (1 mmol) was required. After the addition of $\mathrm{Pd}\left(\mathrm{NH}_{3}\right)_{2} \mathrm{Cl}_{2} / \mathbf{1}$ aqueous solution (in $1 \mathrm{~mL} \mathrm{H} \mathrm{H}_{2} \mathrm{O}$, at different concentrations for various substrate/catalyst ratios), the tube was sealed under air by a Teflon-coated screw cap. The reaction vessel was then placed in an oil bath at $140{ }^{\circ} \mathrm{C}$ for the indicated reaction time period (see Tables 2-4). After cooling of the reaction mixture to room temperature, the aqueous solution was extracted with hexane, the organic phase was dried over $\mathrm{MgSO}_{4}$, and the solvent was then removed under vacuum. Column chromatography on silica gel afforded the desired product.

(E)-3-Phenylacrylic acid n-butyl ester (4a) [40]. Yellow oil. ${ }^{1} \mathrm{H}-\mathrm{NMR}$ : $\delta 0.97(\mathrm{t}, J=7.0 \mathrm{~Hz}, 3 \mathrm{H}$ ), $1.35-1.53(\mathrm{~m}, 2 \mathrm{H}), 1.63-1.77(\mathrm{~m}, 2 \mathrm{H}), 4.21(\mathrm{t}, J=6.6 \mathrm{~Hz}, 2 \mathrm{H}), 6.44(\mathrm{~d}, J=16.0 \mathrm{~Hz}, 1 \mathrm{H}), 7.35-7.41$ $(\mathrm{m}, 3 \mathrm{H}), 7.49-7.56(\mathrm{~m}, 2 \mathrm{H}), 7.67(\mathrm{~d}, J=16.0 \mathrm{~Hz}, 1 \mathrm{H}) ;{ }^{13} \mathrm{C}$ NMR: $\delta$ 13.6, 19.1, 30.7, 64.2, 118.2, $127.8,128.7,129.9,134.4,144.3,166.8$.

(E)-3-(4-Acetylphenyl)acrylic acid n-butyl ester (4b) [41]. Yellow oil. ${ }^{1} \mathrm{H}-\mathrm{NMR}: \delta 0.94$ (t, J= 7.6 Hz, $3 \mathrm{H}), 1.34-1.51(\mathrm{~m}, 2 \mathrm{H}), 1.61-1.75(\mathrm{~m}, 2 \mathrm{H}), 2.60(\mathrm{~s}, 3 \mathrm{H}), 4.21(\mathrm{t}, J=6.4 \mathrm{~Hz}, 2 \mathrm{H}), 6.51$ (d, $J=16.0 \mathrm{~Hz}$, 
1H), $7.59(\mathrm{~d}, J=8.0 \mathrm{~Hz}, 2 \mathrm{H}), 7.67(\mathrm{~d}, J=16.0 \mathrm{~Hz}, 1 \mathrm{H}), 7.95(\mathrm{~d}, J=8.0 \mathrm{~Hz}, 2 \mathrm{H}) ;{ }^{13} \mathrm{C}-\mathrm{NMR}: \delta 13.6$, 19.1, 26.5, 30.7, 64.5, 120.7, 128.0, 128.7, 137.9, 138.7, 142.8, 166.3, 197.0.

(E)-3-(4-Nitrophenyl)acrylic acid n-butyl ester (4c). Yellow solid. Mp. 67-68 (lit.[41] 67-69 $\left.{ }^{\circ} \mathrm{C}\right) .{ }^{1} \mathrm{H}-$ NMR: $\delta 0.95(\mathrm{t}, J=7.4 \mathrm{~Hz}, 3 \mathrm{H}), 1.33-1.51(\mathrm{~m}, 2 \mathrm{H}), 1.61-1.76(\mathrm{~m}, 2 \mathrm{H}), 4.22(\mathrm{t}, J=6.6 \mathrm{~Hz}, 2 \mathrm{H}), 6.54$ $(\mathrm{d}, J=16.0 \mathrm{~Hz}, 1 \mathrm{H}), 7.62-7.73(\mathrm{~m}, 3 \mathrm{H}), 8.23(\mathrm{~d}, J=9.0 \mathrm{~Hz}, 2 \mathrm{H}) ;{ }^{13} \mathrm{C}-\mathrm{NMR}: \delta 13.6,19.1,30.7,64.8$, $122.6,124.0,128.5,140.5,141.4,148.4,165.9$.

(E)-3-(4-Cyanophenyl)acrylic acid n-butyl ester (4d). Yellow solid. Mp. 43.1-43.5 (lit.[42] 43.5-46.9 $\left.{ }^{\circ} \mathrm{C}\right) .{ }^{1} \mathrm{H}-\mathrm{NMR}: \delta 0.95(\mathrm{t}, J=7.2 \mathrm{~Hz}, 3 \mathrm{H}), 1.32-1.51(\mathrm{~m}, 2 \mathrm{H}), 1.61-1.75(\mathrm{~m}, 2 \mathrm{H}), 4.21(\mathrm{t}, J=6.6 \mathrm{~Hz}$, 2H), $6.50(\mathrm{~d}, J=16.2 \mathrm{~Hz}, 1 \mathrm{H}), 7.56-7.68(\mathrm{~m}, 5 \mathrm{H}) ;{ }^{13} \mathrm{C}-\mathrm{NMR}: \delta 13.5,19.0,30.5,64.5,113.1,118.1$, $121.7,128.2,132.4,138.5,141.8,165.9$.

(E)-4-(2-Butoxycarbonylvinyl)benzoic acid ethyl ester (4e). Yellow oil. ${ }^{1} \mathrm{H}-\mathrm{NMR}$ : $\delta 0.97$ (t, $J=7.2 \mathrm{~Hz}$, $3 \mathrm{H}), 1.36-1.57(\mathrm{~m}, 5 \mathrm{H}), 1.63-1.77(\mathrm{~m}, 2 \mathrm{H}), 4.22(\mathrm{t}, J=6.6 \mathrm{~Hz}, 2 \mathrm{H}), 4.39$ (q, $J=7.2 \mathrm{~Hz}, 2 \mathrm{H}), 6.52(\mathrm{~d}$, $J=16.0 \mathrm{~Hz}, 1 \mathrm{H}), 7.58(\mathrm{~d}, J=8.4 \mathrm{~Hz}, 2 \mathrm{H}), 7.69(\mathrm{~d}, J=16.0 \mathrm{~Hz}, 1 \mathrm{H}), 8.06(\mathrm{~d}, J=8.4 \mathrm{~Hz}, 2 \mathrm{H}) ;{ }^{13} \mathrm{C}-$ NMR: $\delta 13.5,14.1,18.9,30.5,60.9,64.3,120.4,127.5,129.7,131.4,138.3,142.8,165.5,166.1$; HRMS calcd. for $\mathrm{C}_{16} \mathrm{H}_{20} \mathrm{O}_{4}, 276.1362$; found, 276.1353.

(E)-3-(4-Chlorophenyl)acrylic acid n-butyl ester (4f) [43]. Colorless oil. ${ }^{1} \mathrm{H}-\mathrm{NMR}: \delta \quad 0.96$ (t, $J=7.4 \mathrm{~Hz}, 3 \mathrm{H}), 1.34-1.53(\mathrm{~m}, 2 \mathrm{H}), 1.62-1.76(\mathrm{~m}, 2 \mathrm{H}), 4.21(\mathrm{t}, J=6.6 \mathrm{~Hz}, 2 \mathrm{H}), 6.41(\mathrm{~d}, J=16.0 \mathrm{~Hz}$, $1 \mathrm{H}), 7.35(\mathrm{~d}, J=8.6 \mathrm{~Hz}, 2 \mathrm{H}), 7.46(\mathrm{~d}, J=8.6 \mathrm{~Hz}, 2 \mathrm{H}), 7.62(\mathrm{~d}, J=16.0 \mathrm{~Hz}, 1 \mathrm{H}) ;{ }^{13} \mathrm{C}-\mathrm{NMR}: \delta 13.5$, $19.9,30.6,64.2,188.7,128.8,128.9,132.8,135.8,142.7,166.3$.

(E)-3-(4-Methoxyphenyl)acrylic acid n-butyl ester (4g) [40]. Yellow oil. ${ }^{1} \mathrm{H}-\mathrm{NMR}$ : $\delta \quad 0.96$ (t, $J=7.2 \mathrm{~Hz}, 3 \mathrm{H}), 1.26-1.53(\mathrm{~m}, 2 \mathrm{H}), 1.62-1.76(\mathrm{~m}, 2 \mathrm{H}), 3.84(\mathrm{~s}, 3 \mathrm{H}), 4.20(\mathrm{t}, J=6.4 \mathrm{~Hz}, 2 \mathrm{H}), 6.31(\mathrm{~d}$, $J=16.0 \mathrm{~Hz}, 1 \mathrm{H}), 6.90(\mathrm{~d}, J=8.6 \mathrm{~Hz}, 2 \mathrm{H}), 7.48(\mathrm{~d}, J=8.6 \mathrm{~Hz}, 2 \mathrm{H}), 7.64(\mathrm{~d}, J=16.0 \mathrm{~Hz}, 1 \mathrm{H}) ;{ }^{13} \mathrm{C}-$ NMR: $\delta 13.7,19.1,30.8,55.2,64.1,114.2,115.7,127.1,129.5,144.1,161.2,167.2$.

(E)-3-(4-Tolyl)acrylic acid n-butyl ester (4h) [41]. Yellow oil. ${ }^{1} \mathrm{H}-\mathrm{NMR}: \delta 0.96(\mathrm{t}, J=7.2 \mathrm{~Hz}, 3 \mathrm{H})$, $1.38-1.53(\mathrm{~m}, 2 \mathrm{H}), 1.62-1.76(\mathrm{~m}, 2 \mathrm{H}), 2.37(\mathrm{~s}, 3 \mathrm{H}), 4.20(\mathrm{t}, J=6.6 \mathrm{~Hz}, 2 \mathrm{H}), 6.39(\mathrm{~d}, J=16.0 \mathrm{~Hz}, 1 \mathrm{H})$, $7.19(\mathrm{~d}, J=8.0 \mathrm{~Hz}, 2 \mathrm{H}), 7.43(\mathrm{~d}, J=8.0 \mathrm{~Hz}, 2 \mathrm{H}), 7.66(\mathrm{~d}, J=16.0 \mathrm{~Hz}, 1 \mathrm{H}) ;{ }^{13} \mathrm{C}-\mathrm{NMR}: \delta 13.5,19.0$, $21.2,30.7,64.0,117.1,127.8,129.3,131.6,140.3,144.2,166.9$.

(E)-3-(4-Hydroxyphenyl)acrylic acid n-butyl ester (4i). Yellow solid. Mp. 74-76 (lit.[44] 72-74 ${ }^{\circ} \mathrm{C}$ ). ${ }^{1} \mathrm{H}-\mathrm{NMR}: \delta 0.94(\mathrm{t}, J=7.2 \mathrm{~Hz}, 3 \mathrm{H}), 1.32-1.50(\mathrm{~m}, 2 \mathrm{H}), 1.63-1.74(\mathrm{~m}, 2 \mathrm{H}), 4.18(\mathrm{t}, J=6.4 \mathrm{~Hz}, 2 \mathrm{H})$, $5.29(\mathrm{~s}, 1 \mathrm{H}), 6.28(\mathrm{~d}, J=16.0 \mathrm{~Hz}, 1 \mathrm{H}), 6.82(\mathrm{~d}, J=8.6 \mathrm{~Hz}, 2 \mathrm{H}), 7.42(\mathrm{~d}, J=8.6 \mathrm{~Hz}, 2 \mathrm{H}), 7.61(\mathrm{~d}$, $J=16.0 \mathrm{~Hz}, 1 \mathrm{H}) ;{ }^{13} \mathrm{C}-\mathrm{NMR}: \delta$ 13.6, 19.1, 30.6, 64.6, 114.5, 116.0, 126.2, 129.9, 145.2, 158.9, 168.5.

(E)-3-(3-Tolyl)acrylic acid n-butyl ester (4j) [41]. Colorless oil. ${ }^{1} \mathrm{H}-\mathrm{NMR}: \delta 0.97(\mathrm{t}, J=7.6 \mathrm{~Hz}, 3 \mathrm{H})$, $1.38-1.54(\mathrm{~m}, 2 \mathrm{H}), 1.62-1.73(\mathrm{~m}, 2 \mathrm{H}), 2.37(\mathrm{~s}, 3 \mathrm{H}), 4.21(\mathrm{t}, J=6.8 \mathrm{~Hz}, 2 \mathrm{H}), 6.42(\mathrm{~d}, J=16.0 \mathrm{~Hz}, 1 \mathrm{H})$, 
7.18-7.35 (m, 4H), $7.65(\mathrm{~d}, J=16.0 \mathrm{~Hz}, 1 \mathrm{H}) ;{ }^{13} \mathrm{C}-\mathrm{NMR}: \delta 13.5,19.0,21.0,30.7,64.0,117.9,124.9$, $128.4,128.4,130.7,134.2,138.1,144.4,166.7$.

(E)-3-(2-Tolyl)acrylic acid n-butyl ester (4k) [45]. Pale yellow oil. ${ }^{1} \mathrm{H}-\mathrm{NMR}: \delta 0.97$ (t, $J=7.2 \mathrm{~Hz}$, $3 \mathrm{H}), 1.38-1.54(\mathrm{~m}, 2 \mathrm{H}), 1.63-1.74(\mathrm{~m}, 2 \mathrm{H}), 2.44(\mathrm{~s}, 3 \mathrm{H}), 4.22(\mathrm{t}, J=6.8 \mathrm{~Hz}, 2 \mathrm{H}), 6.36$ (d, $J=16.0 \mathrm{~Hz}$, 1H), $7.21(\mathrm{~m}, 3 \mathrm{H}), 7.55(\mathrm{~d}, J=7.0 \mathrm{~Hz}, 1 \mathrm{H}), 7.98(\mathrm{~d}, J=16.0 \mathrm{~Hz}, 1 \mathrm{H}) ;{ }^{13} \mathrm{C}-\mathrm{NMR}: \delta 13.7,19.2,19.7$, $30.8,64.4,119.3,126.2,126.4,129.8,130.7,133.4,137.5,142.2,167.0$.

(E)-3-(2-Methoxyphenyl)acrylic acid n-butyl ester (4l) [46]. Yellow oil. ${ }^{1} \mathrm{H}-\mathrm{NMR}: \delta 0.94$ (t, J= 7.4 Hz, $3 \mathrm{H}), 1.36-1.51(\mathrm{~m}, 2 \mathrm{H}), 1.61-1.74(\mathrm{~m}, 2 \mathrm{H}), 3.87(\mathrm{~s}, 3 \mathrm{H}), 4.19(\mathrm{t}, J=6.6 \mathrm{~Hz}, 2 \mathrm{H}), 6.51(\mathrm{~d}, J=16.0 \mathrm{~Hz}$, $1 \mathrm{H}), 6.88-6.98(\mathrm{~m}, 2 \mathrm{H}), 7.28-7.36(\mathrm{~m}, 1 \mathrm{H}), 7.49(\mathrm{~d}, J=7.8 \mathrm{~Hz}, 1 \mathrm{H}), 7.97(\mathrm{~d}, J=16.2 \mathrm{~Hz}, 1 \mathrm{H}) ;{ }^{13} \mathrm{C}-$ NMR: $\delta 13.5,19.0,30.7,55.1,63.9,110.9,118.5,120.4,123.2,128.5,131.1,139.6,158.0,167.2$.

(E)-3-Phenylacrylic acid ethyl ester (5a) [47]. Oil. ${ }^{1} \mathrm{H}-\mathrm{NMR}: \delta 1.32$ (t, $\left.J=7.2 \mathrm{~Hz}, 3 \mathrm{H}\right), 4.25$ (q, $J=7.2 \mathrm{~Hz}, 2 \mathrm{H}), 6.42(\mathrm{~d}, J=16.0 \mathrm{~Hz}, 1 \mathrm{H}), 7.35-7.38(\mathrm{~m}, 3 \mathrm{H}), 7.49-7.53(\mathrm{~m}, 2 \mathrm{H}), 7.67(\mathrm{~d}$, $J=16.0 \mathrm{~Hz}, 1 \mathrm{H}) ;{ }^{13} \mathrm{C}-\mathrm{NMR}: \delta 14.2,60.3,118.2,127.9,128.7,130.0,134.4,144.4,166.8$.

(E)-3-(4-Acetylphenyl)acrylic acid ethyl ester (5b). Yellow solid. Mp. 43-45 ${ }^{\circ} \mathrm{C}$ (lit.[48] 40-42 ${ }^{\circ} \mathrm{C}$ ). ${ }^{1} \mathrm{H}-\mathrm{NMR}: \delta 1.35(\mathrm{t}, J=7.2 \mathrm{~Hz}, 3 \mathrm{H}), 2.62(\mathrm{~s}, 3 \mathrm{H}), 4.28(\mathrm{q}, J=7.2 \mathrm{~Hz}, 2 \mathrm{H}), 6.53(\mathrm{~d}, J=16.0 \mathrm{~Hz}, 1 \mathrm{H})$, $7.61(\mathrm{~d}, J=8.4 \mathrm{~Hz}, 2 \mathrm{H}), 7.70(\mathrm{~d}, J=16.0 \mathrm{~Hz}, 1 \mathrm{H}), 7.97(\mathrm{~d}, J=8.4 \mathrm{~Hz}, 2 \mathrm{H}) ;{ }^{13} \mathrm{C}-\mathrm{NMR}: \delta 14.0,26.3$, $60.4,120.5,127.7,128.5,137.6,138.4,142.6,165.9,196.7$.

(E)-3-(4-Nitrophenyl)acrylic acid ethyl ester (5c). Yellow solid. Mp. 134-136 ${ }^{\circ} \mathrm{C}$ (lit.[49] 132-134 $\left.{ }^{\circ} \mathrm{C}\right) .{ }^{1} \mathrm{H}-\mathrm{NMR}: \delta 1.36(\mathrm{t}, J=7.2 \mathrm{~Hz}, 3 \mathrm{H}), 4.29(\mathrm{q}, J=7.2 \mathrm{~Hz}, 2 \mathrm{H}), 6.56(\mathrm{~d}, J=16.0 \mathrm{~Hz}, 1 \mathrm{H}), 7.67(\mathrm{~d}$, $J=8.8 \mathrm{~Hz}, 2 \mathrm{H}), 7.71(\mathrm{~d}, J=16.0 \mathrm{~Hz}, 1 \mathrm{H}), 8.25(\mathrm{~d}, J=8.8 \mathrm{~Hz}, 2 \mathrm{H}) ;{ }^{13} \mathrm{C}-\mathrm{NMR}: \delta 14.2,60.9,122.5$, $124.0,128.5,140.5,141.4,148.4,165.8$.

(E)-3-(4-Cyanophenyl)acrylic acid ethyl ester (5d). White solid. Mp. $68-70{ }^{\circ} \mathrm{C}$ (lit.[50] 69-69.3 ${ }^{\circ} \mathrm{C}$ ). ${ }^{1} \mathrm{H}-\mathrm{NMR}: \delta 1.32(\mathrm{t}, J=7.2 \mathrm{~Hz}, 3 \mathrm{H}), 4.26(\mathrm{q}, J=7.2 \mathrm{~Hz}, 2 \mathrm{H}), 6.49(\mathrm{~d}, J=16.0 \mathrm{~Hz}, 1 \mathrm{H}), 7.56-7.68$ (m, $5 \mathrm{H}) ;{ }^{13} \mathrm{C}-\mathrm{NMR}: \delta$ 14.2, 60.9, 113.3, 118.2, 121.9, 128.2, 132.5, 138.7, 142.0, 165.9.

(E)-4-(2-Ethoxycarbonylvinyl)benzoic acid ethyl ester (5e). Yellow solid. Mp. $51-53{ }^{\circ} \mathrm{C} .{ }^{1} \mathrm{H}-\mathrm{NMR}$ : $\delta$ $1.35(\mathrm{t}, J=7.2 \mathrm{~Hz}, 3 \mathrm{H}), 1.40(\mathrm{t}, J=7.0 \mathrm{~Hz}, 3 \mathrm{H}), 4.28(\mathrm{q}, J=7.2 \mathrm{~Hz}, 2 \mathrm{H}), 4.39(\mathrm{q}, J=7.0 \mathrm{~Hz}, 2 \mathrm{H})$, $6.52(\mathrm{~d}, J=16.0 \mathrm{~Hz}, 1 \mathrm{H}), 7.58(\mathrm{~d}, J=8.6 \mathrm{~Hz}, 2 \mathrm{H}), 7.70(\mathrm{~d}, J=16.0 \mathrm{~Hz}, 1 \mathrm{H}), 8.05(\mathrm{~d}, J=8.6 \mathrm{~Hz}, 2 \mathrm{H})$; ${ }^{13} \mathrm{C}-\mathrm{NMR}: \delta$ 14.2, 60.6, 61.0, 120.5, 127.7, 129.9, 131.6, 138.4, 143.0, 165.7, 166.3; Anal. calcd. for $\mathrm{C}_{14} \mathrm{H}_{16} \mathrm{O}_{4}$ : C, 67.73; H, 6.50, found C, 67.91; H, 6.64.

(E)-3-(4-Chlorophenyl)acrylic acid ethyl ester (5f) [51]. Oil. ${ }^{1} \mathrm{H}-\mathrm{NMR}: \delta 1.34(\mathrm{t}, J=7.2 \mathrm{~Hz}, 3 \mathrm{H}), 4.27$ $(\mathrm{q}, J=7.2 \mathrm{~Hz}, 2 \mathrm{H}), 6.41(\mathrm{~d}, J=16.0 \mathrm{~Hz}, 1 \mathrm{H}), 7.35(\mathrm{~d}, J=8.6 \mathrm{~Hz}, 2 \mathrm{H}), 7.46(\mathrm{~d}, J=8.6 \mathrm{~Hz}, 2 \mathrm{H}), 7.63$ $(\mathrm{d}, J=16.0 \mathrm{~Hz}, 1 \mathrm{H}) ;{ }^{13} \mathrm{C}-\mathrm{NMR}: \delta 14.3,60.5,118.8,128.6,129.1,132.9,136.0,142.9,166.6$. 
(E)-3-(4-Methoxyphenyl)acrylic acid ethyl ester (5g) [51]. Oil. ${ }^{1} \mathrm{H}-\mathrm{NMR}: \delta 1.33(\mathrm{t}, J=7.2 \mathrm{~Hz}, 3 \mathrm{H}$ ), $3.84(\mathrm{~s}, 3 \mathrm{H}), 4.25(\mathrm{q}, J=7.2 \mathrm{~Hz}, 2 \mathrm{H}), 6.31(\mathrm{~d}, J=16.0 \mathrm{~Hz}, 1 \mathrm{H}), 6.90(\mathrm{~d}, J=8.6 \mathrm{~Hz}, 2 \mathrm{H}), 7.48(\mathrm{~d}$, $J=8.6 \mathrm{~Hz}, 2 \mathrm{H}), 7.64(\mathrm{~d}, J=16.0 \mathrm{~Hz}, 1 \mathrm{H}) ;{ }^{13} \mathrm{C}-\mathrm{NMR}: \delta 14.1,55.0,60.0,114.0,115.5,126.9,129.4$, $143.9,161.1,166.9$.

(E)-3-(4-Tolyl)acrylic acid ethyl ester (5h) [51]. Oil. ${ }^{1} \mathrm{H}-\mathrm{NMR}: \delta 1.34$ (t, J=7.2 Hz, 3H), 2.37 (s, 3H), 4.26 (q, $J=7.2 \mathrm{~Hz}, 2 \mathrm{H}), 6.39$ (d, $J=16.0 \mathrm{~Hz}, 1 \mathrm{H}), 7.19$ (d, $J=8.0 \mathrm{~Hz}, 2 \mathrm{H}), 7.43$ (d, $J=8.0 \mathrm{~Hz}, 2 \mathrm{H}$ ), $7.66(\mathrm{~d}, J=16.0 \mathrm{~Hz}, 1 \mathrm{H}) ;{ }^{13} \mathrm{C}-\mathrm{NMR}: \delta 14.2,21.3,60.2,117.1,127.9,129.4,131.6,140.4,144.4$, 166.9 .

(E)-3-(4-Hydroxyphenyl)acrylic acid ethyl ester (5i) [52]. Oil. ${ }^{1} \mathrm{H}-\mathrm{NMR}: \delta 1.33$ (t, $J=7.2 \mathrm{~Hz}, 3 \mathrm{H}$ ), 4.25 (q, $J=7.2 \mathrm{~Hz}, 2 \mathrm{H}), 6.29$ (d, $J=15.8 \mathrm{~Hz}, 1 \mathrm{H}), 6.84$ (d, $J=8.4 \mathrm{~Hz}, 2 \mathrm{H}), 7.43$ (d, $J=8.4 \mathrm{~Hz}, 2 \mathrm{H})$, $7.63(\mathrm{~d}, J=15.8 \mathrm{~Hz}, 1 \mathrm{H}) ;{ }^{13} \mathrm{C}-\mathrm{NMR}: \delta 14.3,60.4,114.7,116.1,126.1,129.9,144.9,159.2,167.8$.

(E)-3-(3-Tolyl)acrylic acid ethyl ester (5j) [53]. Oil. ${ }^{1} \mathrm{H}-\mathrm{NMR}: \delta 1.34$ (t, $\left.J=7.2 \mathrm{~Hz}, 3 \mathrm{H}\right), 2.37$ (s, 3H), $4.26(\mathrm{q}, J=7.2 \mathrm{~Hz}, 2 \mathrm{H}), 6.42(\mathrm{~d}, J=16.0 \mathrm{~Hz}, 1 \mathrm{H}), 7.17-7.34(\mathrm{~m}, 4 \mathrm{H}), 7.66(\mathrm{~d}, J=16.0 \mathrm{~Hz}, 1 \mathrm{H}) ;{ }^{13} \mathrm{C}-$ NMR: $\delta 14.2,21.2,60.3,117.9,125.1,128.6,128.6,130.9,134.3,138.3,144.6,166.8$.

(E)-3-(2-Tolyl)acrylic acid ethyl ester (5k) [51]. Oil. ${ }^{1} \mathrm{H}-\mathrm{NMR}: \delta 1.35$ (t, $\left.J=7.2 \mathrm{~Hz}, 3 \mathrm{H}\right), 2.45$ (s, 3H), $4.27(\mathrm{q}, J=7.2 \mathrm{~Hz}, 2 \mathrm{H}), 6.36(\mathrm{~d}, J=16.0 \mathrm{~Hz}, 1 \mathrm{H}), 7.21-7.26(\mathrm{~m}, 3 \mathrm{H}), 7.55(\mathrm{~d}, J=7.2 \mathrm{~Hz}, 1 \mathrm{H}), 7.98$ $(\mathrm{d}, J=16.0 \mathrm{~Hz}, 1 \mathrm{H}) ;{ }^{13} \mathrm{C}-\mathrm{NMR}: \delta 14.2,19.6,60.3,119.2,126,2,126.3,129.8,130.6,133.3,137.4$, $142.1,166.8$.

(E)-Stilbene (6a). Yellow solid. Mp. $125-126{ }^{\circ} \mathrm{C}$ (lit.[54] $\left.125{ }^{\circ} \mathrm{C}\right) .{ }^{1} \mathrm{H}-\mathrm{NMR}: \delta 7.11(\mathrm{~s}, 2 \mathrm{H}), 7.29$ (t, $J=6.6 \mathrm{~Hz}, 2 \mathrm{H}), 7.40(\mathrm{t}, J=7.0 \mathrm{~Hz}, 4 \mathrm{H}), 7.53(\mathrm{~d}, J=8.2 \mathrm{~Hz}, 4 \mathrm{H}) ;{ }^{13} \mathrm{C}-\mathrm{NMR}: \delta 126.5,127.6,128.6$, 128.7, 137.3.

(E)-4-Acetylstilbene (6b). White solid. Mp. $145-147{ }^{\circ} \mathrm{C}$ (lit.[55] $148-150{ }^{\circ} \mathrm{C}$ ). ${ }^{1} \mathrm{H}-\mathrm{NMR}$ : $\delta 2.61$ (s, $3 \mathrm{H}), 7.12(\mathrm{~d}, J=16.4 \mathrm{~Hz}, 1 \mathrm{H}), 7.23(\mathrm{~d}, J=16.4 \mathrm{~Hz}, 1 \mathrm{H}), 7.26-7.43(\mathrm{~m}, 3 \mathrm{H}), 7.55(\mathrm{~d}, J=8.0 \mathrm{~Hz}, 2 \mathrm{H})$, 7.59 (d, $J=8.4 \mathrm{~Hz}, 2 \mathrm{H}), 7.95$ (d, $J=8.4 \mathrm{~Hz}, 2 \mathrm{H}) ;{ }^{13} \mathrm{C}-\mathrm{NMR}: \delta 26.6,126.4,126.8,127.4,128.3,128.7$, $128.8,131.4,135.9,136.7,141.9,197.3$.

(E)-4-Nitrostilbene (6c). Yellow solid. Mp. 158-160 ${ }^{\circ} \mathrm{C}$ (lit.[56] $\left.158-158.5{ }^{\circ} \mathrm{C}\right) .{ }^{1} \mathrm{H}-\mathrm{NMR}$ : $\delta 7.14(\mathrm{~d}$, $J=16.2 \mathrm{~Hz}, 1 \mathrm{H}), 7.28(\mathrm{~d}, J=16.2 \mathrm{~Hz}, 1 \mathrm{H}), 7.34-7.45(\mathrm{~m}, 3 \mathrm{H}), 7.55(\mathrm{~d}, J=8.0 \mathrm{~Hz}, 2 \mathrm{H}), 7.64(\mathrm{~d}$, $J=8.0 \mathrm{~Hz}, 2 \mathrm{H}){ }^{13} \mathrm{C}-\mathrm{NMR}: \delta 124.1,126.3,126.8,126.9,128.7,128.8,133.3,136.2,143.8,146.8$.

(E)-4-Cyanostilbene (6d). White solid. Mp. $120{ }^{\circ} \mathrm{C}$ (lit.[55] $\left.117.4-117.7{ }^{\circ} \mathrm{C}\right) .{ }^{1} \mathrm{H}-\mathrm{NMR}: \delta 7.08(\mathrm{~d}$, $J=16.0 \mathrm{~Hz}, 1 \mathrm{H}), 7.22(\mathrm{~d}, J=16.0 \mathrm{~Hz}, 1 \mathrm{H}), 7.32-7.44(\mathrm{~m}, 3 \mathrm{H}), 7.52-7.67(\mathrm{~m}, 6 \mathrm{H}) ;{ }^{13} \mathrm{C}-\mathrm{NMR}: \delta 110.6$, $118.9,126.7,126.8,126.9,128.6,128.8,132.3,132.4,136.3,141.8$.

(E)-4-Ethoxycarbonylstilbene (6e). Colorless solid. Mp 105-107 ${ }^{\circ} \mathrm{C}$ (lit.[57] 106.0-106.5 ${ }^{\circ} \mathrm{C}$ ). ${ }^{1} \mathrm{H}$ NMR: $\delta 1.41(\mathrm{t}, J=7.2 \mathrm{~Hz}, 3 \mathrm{H}), 4.39(\mathrm{q}, J=7.2 \mathrm{~Hz}, 2 \mathrm{H}), 7.12(\mathrm{~d}, J=16.2 \mathrm{~Hz}, 1 \mathrm{H}), 7.23(\mathrm{~d}$, 
$J=16.2 \mathrm{~Hz}, 1 \mathrm{H}), 7.29-7.33(\mathrm{~m}, 1 \mathrm{H}), 7.34-7.43(\mathrm{~m}, 2 \mathrm{H}), 7.53-7.59(\mathrm{~m}, 4 \mathrm{H}), 8.03$ (d, $J=8.4 \mathrm{~Hz}, 2 \mathrm{H})$;

${ }^{13} \mathrm{C}$ - NMR: $\delta$ 14.8, 60.9, 126.2, 126.7, 127.6, 128.2, 128.7, 129.3, 129.9, 131.1, 136.7, 141.7, 166.3 .

(E)-4-Chlorostilbene (6f). White solid. Mp. 130-132 ${ }^{\circ} \mathrm{C}$ (lit.[58] $129{ }^{\circ} \mathrm{C}$ ). ${ }^{1} \mathrm{H}-\mathrm{NMR}: \delta 7.07$ (s, 2H), 7.26-7.41 (m, 5H), $7.44(\mathrm{~d}, J=8.8 \mathrm{~Hz}, 2 \mathrm{H}), 7.50$ (d, $J=7.4 \mathrm{~Hz}, 2 \mathrm{H}) ;{ }^{13} \mathrm{C}-\mathrm{NMR}: \delta 126.5,127.3,127.6$, $127.8,128.7,128.8,129.3,133.1,135.8,136.9$.

(E)-4-Methoxystilbene (6g). Pale white solid. Mp. 135-136 ${ }^{\circ} \mathrm{C}$ (lit.[58] $136{ }^{\circ} \mathrm{C}$ ). ${ }^{1} \mathrm{H}-\mathrm{NMR}$ : $\delta 3.82$ (s, $3 \mathrm{H}), 6.89(\mathrm{~d}, J=8.8 \mathrm{~Hz}, 2 \mathrm{H}), 6.95(\mathrm{~d}, J=16.4 \mathrm{~Hz}, 1 \mathrm{H}), 7.07$ (d, $J=16.4 \mathrm{~Hz}, 1 \mathrm{H}), 7.20-7.22(\mathrm{~m}, 1 \mathrm{H})$, $7.33(\mathrm{t}, J=7.4 \mathrm{~Hz}, 2 \mathrm{H}), 7.41-7.50(\mathrm{~m}, 4 \mathrm{H}) ;{ }^{13} \mathrm{C}-\mathrm{NMR}: \delta 55.3,114.1,126.2,126.6,127.2,127.7$, 128.2, 128.6, 130.2, 137.6, 159.3.

(E)-4-Methylstilbene (6h). Light yellow solid. Mp. 120-121 ${ }^{\circ} \mathrm{C}$ (lit.[58] $121{ }^{\circ} \mathrm{C}$ ). ${ }^{1} \mathrm{H}-\mathrm{NMR}: \delta 2.36$ (s, $3 \mathrm{H}), 7.07(\mathrm{~s}, 2 \mathrm{H}), 7.17(\mathrm{~d}, J=8.0 \mathrm{~Hz}, 2 \mathrm{H}), 7.24-7.26(\mathrm{~m}, 1 \mathrm{H}), 7.35(\mathrm{t}, J=7.4 \mathrm{~Hz}, 2 \mathrm{H}), 7.41(\mathrm{~d}$, $J=8.0 \mathrm{~Hz}, 2 \mathrm{H}), 7.50(\mathrm{~d}, J=7.2 \mathrm{~Hz}, 2 \mathrm{H}) ;{ }^{13} \mathrm{C}-\mathrm{NMR}: \delta 21.3,126.3,126.4,127.4,127.7,128.6,129.4$, $134.5,137.46,137.49$.

(E)-4-Hydroxystilbene (6i). Yellow solid. Mp. 185-187 ${ }^{\circ} \mathrm{C}$ (lit.[59] $\left.186{ }^{\circ} \mathrm{C}\right) .{ }^{1} \mathrm{H}-\mathrm{NMR}: \delta 4.78(\mathrm{~s}, 1 \mathrm{H})$, $6.83(\mathrm{~d}, J=8.8 \mathrm{~Hz}, 2 \mathrm{H}), 6.95(\mathrm{~d}, J=16.4 \mathrm{~Hz}, 1 \mathrm{H}), 7.06(\mathrm{~d}, J=16.4 \mathrm{~Hz}, 1 \mathrm{H}), 7.19-7.26(\mathrm{~m}, 1 \mathrm{H}), 7.32$ $(\mathrm{d}, J=7.6 \mathrm{~Hz}, 2 \mathrm{H}), 7.41(\mathrm{~d}, J=8.6 \mathrm{~Hz}, 2 \mathrm{H}), 7.49(\mathrm{~d}, J=7.2 \mathrm{~Hz}, 2 \mathrm{H}) ;{ }^{13} \mathrm{C}-\mathrm{NMR}: \delta 115.6,126.2$, $126.7,127.2,127.9,128.1,128.6,130.4,137.6,155.2$.

(E)-3-Methylstilbene (6j). Pale yellow solid. Mp. 48-49 ${ }^{\circ} \mathrm{C}$ (lit.[60] 47-48 ${ }^{\circ} \mathrm{C}$ ). ${ }^{1} \mathrm{H}-\mathrm{NMR}: \delta 2.37$ (s, $3 \mathrm{H}), 7.05-7.08(\mathrm{~m}, 3 \mathrm{H}), 7.22-7.28(\mathrm{~m}, 2 \mathrm{H}), 7.29-7.39(\mathrm{~m}, 4 \mathrm{H}), 7.49-7.53(\mathrm{~m}, 2 \mathrm{H}),{ }^{13} \mathrm{C}-\mathrm{NMR}: \delta 24.4$, $123.7,126.5,127.2,127.5,128.4,128.5,128.5,128.6,128.8,137.3,137.4,138.2$.

(E)-2-Methylstilbene (6k) [16]. Oil. ${ }^{1} \mathrm{H}-\mathrm{NMR}: \delta 2.42(\mathrm{~s}, 3 \mathrm{H}), 6.99(\mathrm{~d}, J=16.2 \mathrm{~Hz}, 1 \mathrm{H}), 7.23-7.33$ (m, 4H), 7.37-7.43 (m, 3H), 7.52 (d, $J=7.2 \mathrm{~Hz}, 2 \mathrm{H}), 7.59$ (d, $J=6.8 \mathrm{~Hz}, 1 \mathrm{H}) ;{ }^{13} \mathrm{C}-\mathrm{NMR}: \delta 19.9,125.4$, $126.2,126.5,127.5,127.6,128.6,129.9,130.3,135.7,136.4,137.7$.

(E)-2-Methoxystilbene (6l). Pink solid. Mp. 58-59 ${ }^{\circ} \mathrm{C}$ (lit.[61] 58.6-59.5 $\left.{ }^{\circ} \mathrm{C}\right) .{ }^{1} \mathrm{H}-\mathrm{NMR}: \delta 3.89$ (s, 3H), $6.91(\mathrm{~d}, J=8.4 \mathrm{~Hz}, 1 \mathrm{H}), 6.98(\mathrm{~d}, J=7.4 \mathrm{~Hz}, 1 \mathrm{H}), 7.11(\mathrm{~d}, J=16.6 \mathrm{~Hz}, 1 \mathrm{H}), 7.26-7.43(\mathrm{~m}, 5 \mathrm{H})$, 7.55-7.62 (m, 3H); ${ }^{13} \mathrm{C}$ NMR: $\delta$ 55.5, 110.9, 120.7, 123.5, 126.4, 126.44, 126.5, 127.3, 128.5, 128.6, $129.1,137.9,156.9$.

(E)-2-Hydroxystilbene (6m). Pale white solid. Mp. 144-145 ${ }^{\circ} \mathrm{C}$ (lit.[62] 143-144 $\left.{ }^{\circ} \mathrm{C}\right) .{ }^{1} \mathrm{H}-\mathrm{NMR}: \delta 5.02$ $(\mathrm{s}, 1 \mathrm{H}), 6.81(\mathrm{dd}, J=1.6 \mathrm{~Hz}, J=8.0 \mathrm{~Hz}, 1 \mathrm{H}), 6.95(\mathrm{t}, J=7.4 \mathrm{~Hz}, 1 \mathrm{H}), 7.12(\mathrm{~d}, J=16.6 \mathrm{~Hz}, 1 \mathrm{H}), 7.16$ $(\mathrm{td}, J=1.6 \mathrm{~Hz}, J=8.0 \mathrm{~Hz}, 1 \mathrm{H}), 7.21-7.29(\mathrm{~m}, 1 \mathrm{H}), 7.37(\mathrm{~d}, J=16.6 \mathrm{~Hz}, 1 \mathrm{H}), 7.32-7.42(\mathrm{~m}, 2 \mathrm{H})$, 7.51-7.55 (m, 3H); ${ }^{13} \mathrm{C}-\mathrm{NMR}: \delta 115.9,121.1,123.0,124.7,126.5,127.3,127.6,128.6,130.2,137.6$, 152.9 . 


\subsection{Typical procedure for the reuse of the catalytic aqueous solution}

The reaction was conducted following the procedure described in Section 3.2 under the reaction conditions shown in Table 5. After reaction, the aqueous reaction mixture was washed with hexane under vigorous stirring three times, and the organic product was isolated from the combined organic phase according to the previously-described procedure. The residual aqueous solution was then charged with aryl iodide, alkene, and $\mathrm{Bu}_{3} \mathrm{~N}$ for the next reaction, and in the case of styrene, addition of $\mathrm{TBAB}$ at the first run was required.

\section{Conclusions}

In conclusion, we have shown here that the above-described $\mathrm{Pd}\left(\mathrm{NH}_{3}\right)_{2} \mathrm{Cl}_{2}$ /cationic 2,2'-bipyridyl system could be a highly-efficient catalyst for the Mizoroki-Heck coupling of aryl iodides and alkenes using an environmentally benign solvent, water, as the reaction medium. The loading amount of the catalyst in a single batch reaction can be reduced to as low as $0.0001 \mathrm{~mol} \%$, while still affording the products in high yields. This water-compatible and air-stable catalytic system enables the reaction to be conducted using a very simple procedure. The catalyst can be easily separated from the organic products by simple extraction and the residual aqueous solution can be reused for further reactions, which reduces the wastage of precious metal, making this procedure greener and economically viable.

\section{Acknowledgements}

This research was financially supported by the National Science Council of Taiwan (NSC96-2113M-027-003-MY2).

\section{References}

1. Mizoroki, T.; Mori, K.; Ozaki, A. Arylation of olefin with aryl iodide catalyzed by palladium. Bull. Chem. Soc. Jpn. 1971, 44, 581-581.

2. Heck, R.F.; Nolley, J.P., Jr. Palladium-Catalyzed vinylic hydrogen substitution reactions with aryl, benzyl, and styryl halides. J. Org. Chem. 1972, 37, 2320-2322.

3. Nicolaou, K.C.; Bulger, P.G.; Sarlah, D. Palladium-catalyzed cross-coupling reactions in total synthesis. Angew. Chem. Int. Ed. 2005, 44, 4442-4489.

4. Dounay, A.B.; Overman, L.E. The asymmetric intramolecular Heck reaction in natural product total synthesis. Chem. Rev. 2003, 103, 2945-2964.

5. Whitcombe, N.J.; Hii, K.K.; Gibson, S.E. Advances in the Heck chemistry of aryl bromides and chlorides. Tetrahedron 2001, 57, 7449-7476.

6. Beletskaya, I.P.; Cheprakov. A.V. The Heck reaction as a sharpening stone of palladium catalysis. Chem. Rev. 2000, 100, 3009-3066.

7. Shaughnessy, K.H. Hydrophilic ligands and their application in aqueous-phase metal-catalyzed reactions. Chem. Rev. 2009, 109, 643-710.

8. Horváth, I.T. Solvents from nature. Green Chem. 2008, 10, 1024-1028.

9. Li, C.J.; Trost, B.M. Green chemistry for chemical synthesis. Proc. Natl. Acad. Sci. USA 2008, 105, 13197-13202. 
10. Liu, S.; Xiao, J. Toward green catalytic synthesis-Transition metal-catalyzed reactions in nonconventional media. J. Mol. Catal. A Chem. 2007, 270, 1-43.

11. Chen, L.; Li, C.J. Catalyzed reactions of alkynes in water. Adv. Synth. Catal. 2006, 348, 14591484.

12. Li, C.J.; Chen, L. Organic chemistry in water. Chem. Soc. Rev. 2006, 35, 68-82.

13. Sheldon, R.A. Green solvents for sustainable organic synthesis: State of the art. Green Chem. 2005, 7, 267-278.

14. Li, C.J. Organic Reactions in aqueous media with a focus on carbon-carbon bond formations: A decade update. Chem. Rev. 2005, 105, 3095-3166.

15. Jeffery, T. On the efficiency of tetraalkylammonium salts in Heck type reactions. Tetrahedron 1996, 52, 10113-10130.

16. Arvela, R.K.; Leadbeater, N.E. Microwave-promoted Heck coupling using ultralow metal catalyst concentrations. J. Org. Chem. 2005, 70, 1786-1790.

17. Solabannavar, S.B.; Desai, U.V.; Mane, R.B. Heck reaction in aqueous medium using Amberlite IRA-400 (basic). Green Chem. 2002, 4, 347-348.

18. Kumar, N.S.C.R.; Raj, I.V.P.; Sudalai, A. Sulfonamide- and hydrazine-based palladium catalysts: Stable and efficient catalysts for $\mathrm{C}-\mathrm{C}$ coupling reactions in aqueous medium. J. Mol. Catal. A Chem. 2007, 269, 218-224.

19. Dawood, K.M. Microwave-assisted Suzuki-Miyaura and Heck-Mizoroki cross-coupling reactions of aryl chlorides and bromides in water using stable benzothiazole-based palladium(II) precatalysts. Tetrahedron 2007, 63, 9642-9651.

20. Uozumi, Y.; Watanabe, T. Green catalysis: hydroxycarbonylation of aryl halides in water catalyzed by an amphiphilic resin-supported phosphine-palladium complex. J. Org. Chem. 1999, 64, 6921-6923.

21. Arvela, R.K.; Pasquini, S.; Larhed, M. Highly regioselective internal Heck arylation of hydroxyalkyl vinyl ethers by aryl halides in water. J. Org. Chem. 2007, 72, 6390-6396.

22. Botella, L.; Nájera, C. Mono- and $\beta, \beta$-double-Heck reactions of $\alpha, \beta$-unsaturated carbonyl compounds in aqueous media. J. Org. Chem. 2005, 70, 4360-4369.

23. Gülcemal, S.; Kahraman, S.; Daran, J.C.; Çetinkaya, E.; Çetinkaya, B. The synthesis of oligoether-substituted benzimidazolium bromides and their use as ligand precursors for the Pdcatalyzed Heck coupling in water. J. Organomet. Chem. 2009, 694, 3580-3589.

24. Pawar, S.S.; Dekhane, D.V.; Shingare, M.S.; Thore, S.N. Sodium 2-(2-pyridin-3ylethylamino)sulfonate: An efficient ligand and base for palladium-catalyzed Heck reaction in aqueous media. Tetrahedron Lett. 2008, 49, 4252-4255.

25. Mukhopadhyay, S.; Rothenberg, G.; Joshi, A.; Baidossi, M.; Sasson, Y. Heterogeneous palladium-catalysed Heck reaction of aryl chlorides and styrene in water under mild conditions. Adv. Synth. Catal. 2002, 344, 348-354.

26. Hagiwara, H.; Sugawara, Y.; Hoshi, T.; Suzuki, T. Sustainable Mizoroki-Heck reaction in water: Remarkably high activity of $\mathrm{Pd}(\mathrm{OAc})_{2}$ immobilized on reversed phase silica gel with the aid of an ionic liquid. Chem. Commun. 2005, 2942-2944. 
27. Schönfelder, D.; Nuyken, O.; Weberskirch, R. Heck and Suzuki coupling reactions in water using poly(2-oxazoline)s functionalized with palladium carbene complexes as soluble, amphiphilic polymer supports. J. Organomet. Chem. 2005, 690, 4648-4655.

28. Zheng, P.; Zhang, W. Synthesis of efficient and reusable palladium catalyst supported on $\mathrm{pH}-$ responsive colloid and its application to Suzuki and Heck reaction in water. J. Catal. 2007, 250, 324-330.

29. Xu, Y.; Zhang, L.; Cui, Y. Catalytic performance of cellulose supported palladium complex for Heck reaction in water. J. Appl. Polym. Sci. 2008, 110, 2996-3000.

30. Wan, Y.; Wang, H.; Zhao, Q.; Klingstedt, M.; Terasaki, O.; Zhao, D. Ordered mesoporous $\mathrm{Pd} /$ silica-carbon as a highly active heterogeneous catalyst for coupling reaction of chlorobenzene in aqueous media. J. Am. Chem. Soc. 2009, 131, 4541-4550.

31. Firouzabadi, H.; Iranpoor, N.; Gholinejad, M. 2-Aminophenyl diphenylphosphinite as a new ligand for heterogeneous palladium-catalyzed Heck-Mizoroki reactions in water in the absence of any organic co-solvent. Tetrahedron 2009, 65, 7079-7084.

32. Qiao, K.; Sugimura, R.; Bao, Q.; Tomida, D.; Yokoyama, C. An efficient Heck reaction in water catalyzed by palladium nanoparticles immobilized on imidazolium-styrene copolymers. Catal. Commun. 2008, 9, 2470-2474.

33. Senra, J.D.; Malta, L.F.B.; da Costa, M.E.H.M.; Michel, R.C.; Aguiar, L.C.S.; Simas, A.B.C.; Antunes, O.A.C. Hydroxypropyl- $\alpha$-cyclodextrin-capped palladium nanoparticles: active scaffolds for efficient carbon-carbon bond forming cross-couplings in water. Adv. Synth. Catal. 2009, 351, 2411-2422.

34. Sawoo, S.; Srimani, D.; Dutta, P.; Lahiri, R.; Sarkar, A. Size controlled synthesis of Pd nanoparticles in water and their catalytic application in $\mathrm{C}-\mathrm{C}$ coupling reactions. Tetrahedron 2009, 65, 4367-4374.

35. Wu, W.Y.; Chen, S.N.; Tsai, F.Y. Recyclable and highly active cationic 2,2'-bipyridyl palladium(II) catalyst for Suzuki cross-coupling reaction in water. Tetrahedron Lett. 2006, 47, 9267-9270.

36. Chen, S.N.; Wu, W.Y.; Tsai, F.Y. Hiyama reaction of aryl bromides with arylsiloxanes catalyzed by a reusable palladium(II)/cationic bipyridyl system in water. Tetrahedron 2008, 64, 8164-8168.

37. Chen, S.N.; Wu, W.Y.; Tsai, F.Y. Homocoupling reaction of terminal alkynes catalyzed by a reusable cationic 2,2'-bipyridyl palladium(II)/CuI system in water. Green Chem. 2009, 11, 269-274.

38. Wang, Y.H.; Tsai, F.Y. Reusable rhodium(I)/cationic bipyridyl-catalyzed polymerization of phenylacetylenes in water under aerobic conditions. Chem. Lett. 2007, 1492-1493.

39. Wu, W.Y.; Wang, J.C.; Tsai, F.Y. A reusable $\mathrm{FeCl}_{3} \cdot 6 \mathrm{H}_{2} \mathrm{O}$ /cationic 2,2'-bipyridyl catalytic system for the coupling of aryl iodides with thiols in water under aerobic conditions. Green Chem. 2009, $11,326-329$.

40. Cui, X.; Li, Z.; Tao, C.Z.; Xu, Y.; Li, J.; Liu, L.; Guo, Q.X. N,N-Dimethyl- $\beta$-alanine as an inexpensive and efficient ligand for palladium-catalyzed Heck reaction. Org. Lett. 2006, 8, 2467-2470.

41. Mino, T.; Shirae, Y.; Sasai, Y.; Sakamoto, M.; Fujita, T. Phosphine-free palladium catalyzed Mizoroki-Heck reaction using hydrazone as a ligand. J. Org. Chem. 2006, 71, 6834-6839. 
42. Aksın, Ö.; Türkmen, H.; Artok, L.; Çetinkaya, B.; Ni, C.; Büyükgüngör, O.; Özkal, E. Effect of immobilization on catalytic characteristics of saturated Pd-N-heterocyclic carbenes in MizorokiHeck reactions. J. Organomet. Chem. 2006, 691, 3027-3036.

43. Chen, T.; Gao, J.; Shi, M. A novel tridentate NHC-Pd(II) complex and its application in the Suzuki and Heck-type cross-coupling reactions. Tetrahedron 2006, 62, 6289-6294.

44. Venkateswarlu, S.; Ramachandra, M.S.; Krishnaraju, A.V.; Trimurtulu, G.; Subbaraju,G.V. Antioxidant and antimicrobial activity evaluation of polyhydroxycinnamic acid ester derivatives. Indian J. Chem. Sect B 2006, 45, 252-257.

45. Kondolff, I.; Feuerstein, M.; Doucet, H.; Santelli, M. Synthesis of all-cis-3-(2diphenylphosphinoethyl)-1,2,4-tris(diphenylphosphinomethyl)cyclopentane (Ditricyp) from dicyclopentadiene. Tetrahedron 2007, 63, 9514-9521.

46. Andappan, M.M.S.; Nilsson, P.; Larhed, M. Arylboronic acids as versatile coupling partners in fast microwave promoted oxidative Heck chemistry. Mol. Divers. 2003, 7, 97-106.

47. Zeitler, K. Stereoselective synthesis of $(E)-\alpha, \beta$-unsaturated esters via carbene-catalyzed redox esterification. Org. Lett. 2006, 8, 637-640.

48. Moreno-Mañas, M. Pérez, M.; Pleixats, R. Stereospecific preparation of ethyl (E) and (Z)-3-aryl3-phenylpropenoates by Heck reaction. Tetrahedron Lett. 1996, 37, 7449-7452.

49. Masllorens, J.; Moreno-Mañas, M.; Pla-Quintana, A. Roglans, A. First Heck reaction with arenediazonium cations with recovery of Pd-triolefinic macrocyclic catalyst. Org. Lett. 2003, 5, $1559-1561$.

50. Mu, B.; Li, T.; Xu, W.; Zeng, G.; Liu, P.; Wu, Y. Synthesis, characterization, and applications in Heck and Suzuki coupling reactions of amphiphilic cyclopalladated ferrocenylimines. Tetrahedron 2007, 63, 11475-11488.

51. Chen, Y.; Huang, L.; Ranade, M.A.; Zhang, X.P. Iron(III) and ruthenium(II) porphyrin complexcatalyzed selective olefination of aldehydes with ethyl diazoacetate. J. Org. Chem. 2003, 68, 3714-3717.

52. Buchynskyy, A.; Stembera, K.; Hennig, L.; Findeisen, M.; Giesa, S.; Welzel, P. A Method for the introduction of reporter groups into moenomycin A, based on thiouronium salt chemistry. Eur. J. Org. Chem. 2002, 1163-1174.

53. Zhou, P.; Li, Y.; Sun, P.; Zhou, J.; Bao, J. A novel Heck reaction catalyzed by Co hollow nanospheres in ligand-free condition. Chem. Commun. 2007, 1418-1420.

54. Li, J.H.; Li, J.L.; Wang, D.P.; Pi, S.F.; Xie, Y.X.; Zhang, M.B.; Hu, X.C. CuI-catalyzed Suzuki-Miyaura and Sonogashira cross-coupling reactions using DABCO as ligand. J. Org. Chem. 2007, 72, 2053-2057.

55. Iyer, S.; Kulkarni, G.M.; Ramesh, C. Mizoroki-Heck reaction, catalysis by nitrogen ligand Pd complexes and activation of aryl bromides. Tetrahedron 2004, 60, 2163-2172.

56. Hunter, D.H.; Cram, D.J. Electrophilic substitution at saturated carbon. XXVIII. The stereochemical capabilities of vinyl anions ${ }^{1}$. J. Am. Chem. Soc. 1966, 88, 5765-5776.

57. Nakao, Y.; Imanaka, H.; Chen, J.; Yada, A.; Hiyama, T. Synthesis and cross-coupling reaction of alkenyl[(2-hydroxymethyl)phenyl]dimethylsilanes. J. Organomet. Chem. 2007, 692, 585-603.

58. Wheeler, O.H.; de Pabon. H.N.B. Synthesis of stilbenes. A comparative study ${ }^{1}$. J. Org. Chem. 1965, 30, 1473-1477. 
59. Güsten, H.; Salzwedel, M. Die kernmagnetischen resonanzpektren substituierter cis-stilbene. Tetrahedron 1967, 23, 173-185.

60. Wang, L.; Li, H.; Li, P. Task-specific ionic liquid as base, ligand and reaction medium for the palladium-catalyzed Heck reaction. Tetrahedron 2009, 65, 364-368.

61. Wood, C.S.; Mallory, F.B. Photochemistry of stilbenes. IV. The preparation of substituted phenanthrenes $^{1 \mathrm{a}-\mathrm{c}}$. J. Org. Chem. 1964, 29, 3373-3377.

62. Truce, W.E.; Lin, C.I.M. Stereoselective sulfene-tropone cycloadditions and stereospecific thermolysis of resulting adducts. J. Am. Chem. Soc. 1973, 95, 4426-4428.

Sample Availability: Samples of the compounds are available from the authors.

(C) 2010 by the authors; licensee, Molecular Diversity Preservation International, Basel, Switzerland. This article is an open-access article distributed under the terms and conditions of the Creative Commons Attribution license (http://creativecommons.org/licenses/by/3.0/). 